(1)

CrossMark

\title{
Effect of obstructive sleep apnoea on severity and short-term prognosis of acute coronary syndrome
}

\author{
Ferran Barbé ${ }^{1,2}$, Alicia Sánchez-de-la-Torre ${ }^{1,2}$, Jorge Abad ${ }^{3}$, \\ Joaquin Durán-Cantolla ${ }^{2,4}$, Olga Mediano ${ }^{5}$, Jose Amilibia ${ }^{6}$, Maria José Masdeu ${ }^{7}$, \\ Marina Florés ${ }^{1}$, Antonia Barceló ${ }^{2,8}$, Mónica de la Peña ${ }^{2,8}$, Albina Aldomá9 \\ Fernando Worner ${ }^{9}$, Joan Valls ${ }^{10}$, Gerard Castellà ${ }^{10}$ and \\ Manuel Sánchez-de-la-Torre ${ }^{1,2}$ on behalf of the Spanish Sleep Network
}

Affiliations: ${ }^{1}$ Respiratory Dept, Hosp Universitari Arnau de Vilanova and Santa Maria, IRBLleida, Lleida, Spain. ${ }^{2}$ Centro de Investigación Biomédica en Red de Enfermedades Respiratorias (CIBERES), Madrid, Spain. ${ }^{3}$ Respiratory Dept, Hosp Universitari Germans Trias I Pujol, Badalona, Barcelona, Spain. ${ }^{4}$ Bio-Araba Research Institute, Araba University Hospital, Dept of Medicine of Basque Country University, Vitoria-Gasteiz, Spain. ${ }^{5}$ Respiratory Dept, Hospital Universitario de Guadalajara, Guadalajara, Spain. ${ }^{6}$ Respiratory Dept, Hospital Universitario Cruces, Bilbao, Spain. ${ }^{7}$ Respiratory Dept, Hospital Parc Tauli, Sabadell, Barcelona, Spain. ${ }^{8}$ Clinic Analysis and Respiratory Services, Hospital Universitari Son Espases, Institut de investigació sanitaria de Palma (IdisPa), Palma de Mallorca, Spain. ${ }^{9}$ Cardiology Dept, Hospital Universitari Arnau de Vilanova, IRBLleida, Lleida, Spain. ${ }^{10}$ Dept of Statistics, IRB Lleida, Lleida, Spain.

Correspondence: Ferran Barbé, Respiratory Dept, Hospital Univ Arnau de Vilanova, Rovira Roure, 80, 25198 Lleida, Spain. E-mail: febarbe.lleida.ics@gencat.cat

ABSTRACT The goal of this study was to evaluate the influence of obstructive sleep apnoea on the severity and short-term prognosis of patients admitted for acute coronary syndrome.

Obstructive sleep apnoea was defined as an apnoea-hypopnoea index (AHI) $>15 \mathrm{~h}^{-1}$. We evaluated the acute coronary syndrome severity (ejection fraction, Killip class, number of diseased vessels, and plasma peak troponin) and short-term prognosis (length of hospitalisation, complications and mortality).

We included 213 patients with obstructive sleep apnoea (mean \pm sD AHI $30 \pm 14 \mathrm{~h}^{-1}, 61 \pm 10$ years, $80 \%$ males) and 218 controls (AHI $6 \pm 4 \mathrm{~h}^{-1}, 57 \pm 12$ years, $82 \%$ males). Patients with obstructive sleep apnoea exhibited a higher prevalence of systemic hypertension ( $55 \%$ versus $37 \%, \mathrm{p}<0.001)$, higher body mass index $\left(29 \pm 4 \mathrm{~kg} \cdot \mathrm{m}^{-2}\right.$ versus $\left.26 \pm 4 \mathrm{~kg} \cdot \mathrm{m}^{-2}, \mathrm{p}<0.001\right)$, and lower percentage of smokers $(61 \%$ versus $71 \%$, $\mathrm{p}=0.04)$. After adjusting for smoking, age, body mass index and hypertension, the plasma peak troponin levels were significantly elevated in the obstructive sleep apnoea group $\left(831 \pm 908 \mathrm{ng} \cdot \mathrm{L}^{-1}\right.$ versus $987 \pm 884 \mathrm{ng} \cdot \mathrm{L}^{-1}$, $\mathrm{p}=0.03$ ) and higher AHI severity was associated with an increased number of diseased vessels $(\mathrm{p}=0.04)$. The mean length of stay in the coronary care unit was higher in the obstructive sleep apnoea group $(\mathrm{p}=0.03)$.

This study indicates that obstructive sleep apnoea is related to an increase in the peak plasma troponin levels, number of diseased vessels, and length of stay in the coronary care unit.

@ERSpublications

OSA is associated with an increase in the severity of acute coronary syndrome and extended coronary unit stay http://ow.ly/FEsBA

This article has supplementary material available from erj.ersjournals.com

Received: April 162014 | Accepted after revision: Oct 022014

Support statement: Supported by ResMed Ltd. (Australia), Fondo de Investigación Sanitaria (PI10/02763 and PI10/ 02745), the Spanish Respiratory Society (SEPAR), the Catalonian Cardiology Society, Esteve-Teijin (Spain), Oxigen Salud (Spain), ALLER and Neumomadrid.

Conflict of interest: Disclosures can be found alongside the online version of this article at erj.ersjournals.com

Copyright $\odot$ ERS 2015 


\section{Introduction}

Obstructive sleep apnoea (OSA) is a common disease that affects $3-7 \%$ of the general population $[1,2]$. Sleep apnoea is caused by the collapse of the upper airway during sleep, which leads to transient asphyxia. OSA is associated with an increased risk of fatal and nonfatal cardiovascular events [3] and effective continuous positive airway pressure (CPAP) treatment reduces the incidence of hypertension and cardiovascular events [4]. The consequences of OSA are largely mediated by chronic intermittent hypoxia and sleep fragmentation, which may contribute to the pathogenesis of cardiovascular disease in patients with sleep apnoea [5]. Intermittent episodes of hypoxia and arousal cause an increase in sympathetic activity [6] and sudden changes in systemic blood pressure [7], which contribute to the development of myocardial hypertrophy, cardiac arrhythmias and death. OSA is associated with abnormalities in cardiac autonomic and electrophysiological factors, including heart rate variability, the duration of the QT interval, baroreflex function, and chemoreceptor sensitivity. Serious and potentially fatal arrhythmias occur during sleep in patients with OSA [8] and are attenuated by effective treatment with CPAP [9]. Subjects with OSA are at increased risk of sudden death from cardiac causes during sleeping hours [10].

These pathogenic factors in OSA patients may play an aggravating role in the severity of acute myocardial infarction (AMI) and the short-term prognosis of patients. Nevertheless, a cardioprotective role of OSA in the context of AMI, via ischaemic preconditioning, has also been postulated. This latter hypothesis implies the activation of adaptive mechanisms, such as the increased recruitment of proliferative and angiogenic endothelial progenitor cells [11]. Furthermore, patients with OSA reportedly exhibit less severe cardiac injury during an acute nonfatal myocardial infarction compared with patients without OSA [12].

Due to this contradictory evidence, we performed an observational study to evaluate the impact of OSA on the severity and short-term prognosis of patients with acute coronary syndrome (ACS). The objective of the study was to compare the ejection fraction, Killip class, number of diseased vessels, peak troponin, length of hospitalisation, number of complications, and mortality rate of a cohort of ACS patients with and without OSA.

\section{Methods}

Patients

This is an ancillary study of the ISAACC study (a randomised trial of NCT01335087 (Continuous Positive Airway Pressure (CPAP) in Patients With Acute Coronary Syndrome and Obstructive Sleep Apnea (OSA) (ISAACC)), which is a multicentre, open-label, parallel, prospective, randomised, controlled trial [13]. The ISAACC study evaluates the effect of CPAP treatment on the incidence of new cardiovascular events in patients with an episode of ACS and OSA. The ISAACC study includes nonsleepy patients because it is unethical to fail to treat OSA patients with excessive daytime sleepiness. We evaluated patients who were admitted for ACS to coronary care units or cardiology hospitalisation wards at fourteen teaching hospitals in Spain (male and females aged $\geqslant 18$ years). Those patients who met the inclusion criteria and did not meet any of the exclusion criteria underwent respiratory polygraphy during the first $48-72 \mathrm{~h}$ after admission. Patients with an apnoea-hypopnoea index (AHI) $>15$ events $^{-1}$ were randomised to conservative or CPAP treatment. Those patients with an $\mathrm{AHI} \leqslant 15$ events $\cdot \mathrm{h}^{-1}$ were considered controls. Because we expected that $70 \%$ of the patients who underwent respiratory polygraphy would exhibit an $\mathrm{AHI} \leqslant 15$ events. $\mathrm{h}^{-1}$, we randomly excluded two thirds of these patients to guarantee the same rate of inclusion as in the OSA group. It was performed using a computer-generated random numbers list. For the current study, we compared the short-term prognosis and severity of the ACS for patients included in the conservative arm versus the control group (fig. 1).

Acute coronary syndrome was defined as the acute presentation of coronary disease with or without ST elevation infarction, unstable angina, or type 1 MI [14]. The exclusion criteria included the following: previous treatment with CPAP; psychophysical inability to complete questionnaires; the presence of any previously diagnosed sleep disorder; patients with $>50 \%$ central apnoeas or the presence of Cheyne-Stokes respiration; daytime sleepiness (Epworth Sleepiness Scale (ESS) >10); patients with chronic diseases, e.g. neoplasms, renal insufficiency (GFR $<15 \mathrm{~mL} \cdot \mathrm{min}^{-1} \cdot 1.73 \mathrm{~m}^{-2}$ ), severe chronic obstructive pulmonary disorder (a forced expiratory volume in $1 \mathrm{~s}<50 \%$ ), chronic depression, and other limiting chronic diseases; a medical history that could interfere with the study objectives; any processes, whether cardiovascular or otherwise, that reduce life expectancy to $<1$ year; and patients in cardiogenic shock.

The ethics committee of each participating centre approved the study (approval number in the coordinator centre: 2010-852), and patients provided written informed consent.

\section{Procedures}

The diagnosis was based on the results of overnight cardio-respiratory polygraphy, which is in accordance with the guidelines of the Spanish national consensus on the apnoea-hypopnoea syndrome [15]. 


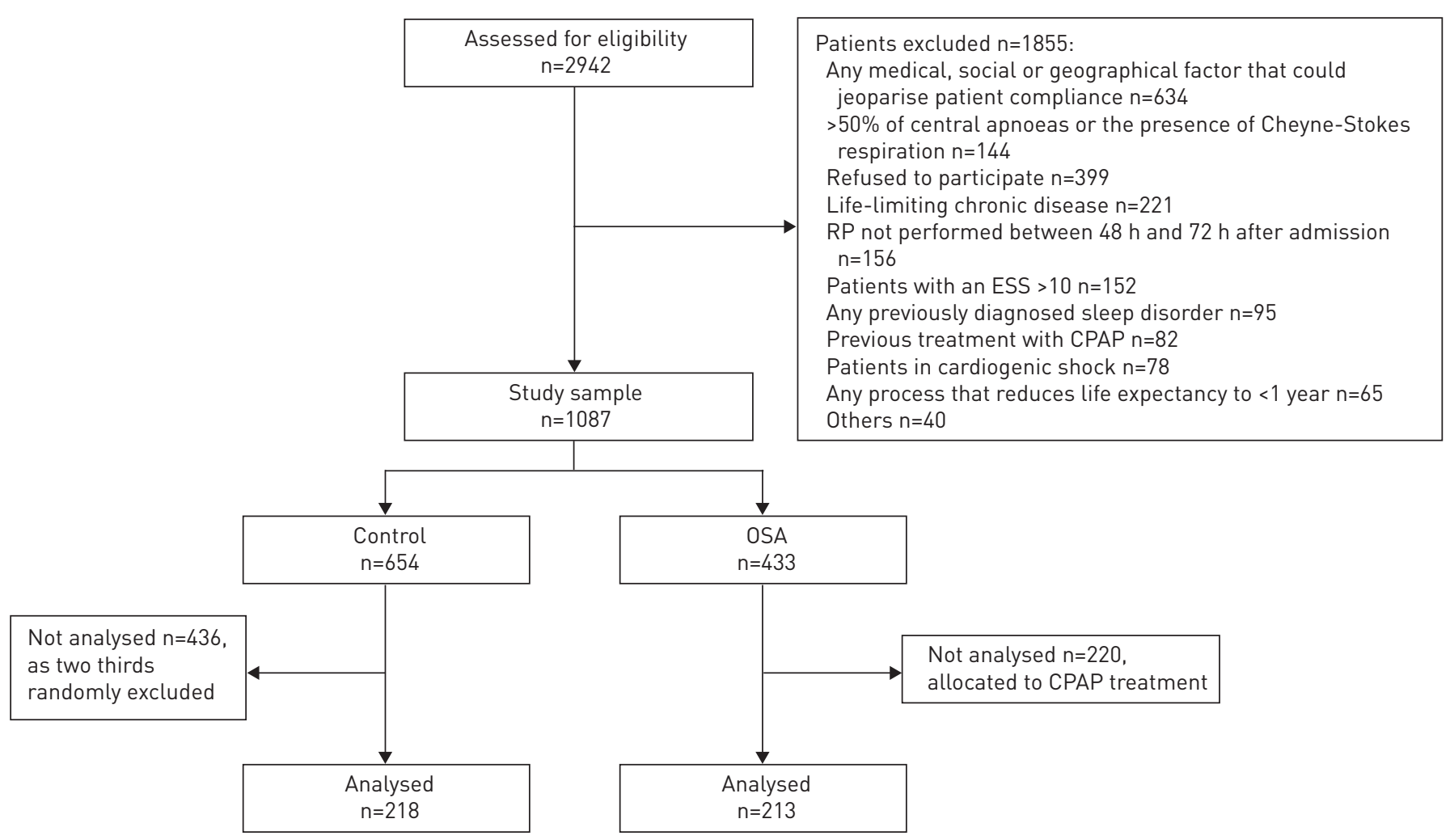

FIGURE 1 Study flowchart showing recruitment to study. RP: respiratory polygraphy; ESS: Epworth Sleepiness Scale; CPAP: continuous positive airway pressure; OSA: obstructive sleep apnoea.

All participant centres used the same model of polygraph (Embletta; ResMed, Bella Vista, Australia) for the diagnosis of OSA. Oronasal flow, thoracoabdominal movements, ECG, and pulse oximetry were recorded. Apnoea was defined as an absence of airflow lasting $\geqslant 10 \mathrm{~s}$. Hypopnoea was defined as a reduction in airflow lasting $\geqslant 10 \mathrm{~s}$ associated with oxygen desaturation. Oxygen desaturation was defined as a decrease in arterial oxygen saturation $>4 \%$. Respiratory polygraphy studies were performed without supplemental oxygen. The AHI was defined as the number of episodes of apnoea and hypopnoea per hour of recording. The degree of self-reported sleepiness/drowsiness was analysed by the Spanish version of the ESS test [16]. Echocardiographic evaluation and Killip classification were routinely performed during patient admission. The Killip classification focuses on physical examination and the development of heart failure to predict risk. The classification considers four classes (I-IV). Class I indicates no evidence of heart failure, and Class IV represents cardiogenic shock. During hospitalisation, we evaluated the severity of the ACS (ejection fraction, the Killip scale, number of affected vessels, number of stents implanted and peak troponin) and the short-term prognosis (length of stay in the coronary unit, length of hospitalisation, complications and mortality). The cardiologists who evaluated the ACS severity were blinded to OSA versus control status.

\section{Data analysis}

Data for each participant were uploaded to a database. Only the coordinating centre (Hospital Univ. Arnau de Vilanova and Santa Maria, IRBLeida, Lleida, Spain) had full access to the database. The mean \pm SD or frequencies (\%) were computed to evaluate the differences between the control and OSA patients with respect to anthropometric and clinical variables and ACS related risk factors, assessing the significance with Mann-Whitney or Chi-squared tests, respectively. Secondly, the association between OSA and variables related to ACS severity and short-term prognosis were assessed with the Mann-Whitney or Chi-squared tests and linear or logistic regression models, as appropriate. Additionally, the models were adjusted for tobacco (current or former smoker versus nonsmoker), age, body mass index (BMI) and hypertension. To evaluate the difference in the mortality observed in patients with and without OSA, an exact test, based on independent binomial distributions, was used given that only one case was observed. Thirdly, the association of the degree of OSA with variables related to ACS severity and short-term prognosis was also assessed using AHI quartiles (Q) and the Kruskal-Wallis or Chi-squared tests and linear and logistic regression models, adjusting for tobacco (current or former smoker versus nonsmoker), age, BMI and 
hypertension. For these analyses, p-values from ANOVA or deviance tables were calculated considering the four AHI-based groups as qualitative and ordinal integer variables. Logarithmic transformation of continuous response variables was performed for the models, assessing the log-likelihood of this transformation with a Box-Cox analysis. For ordinal qualitative variables, integer values were used to perform a linear regression model to assess the trend. Finally, for those variables related to ACS severity with a significant difference between control and OSA patients, a Gini criterion was used to determine an optimal threshold. Odds ratios, corresponding 95\% confidence intervals, and p-values estimated from logistic regression models were obtained; adjusted p-values were also computed. All analyses were performed using the R statistical package [17]. The threshold for significance was set at 0.05 .

\section{Sample size}

The sample sizes, of 213 patients with OSA and 218 controls, provided a statistical power of $84 \%$ to detect differences in the mean length of stay in the coronary care unit and a statistical power of $87 \%$ to detect differences in the distribution of plasma peak troponin levels, when compared by nonparametric tests. The significance level was fixed at 0.05 .

\section{Results}

We included 218 patients with an AHI $\leqslant 15$ events $\cdot \mathrm{h}^{-1}$ (control group) and 213 patients with an AHI >15 events.h ${ }^{-1}$ (OSA group). OSA patients were slightly, but significantly, older than the controls (table 1)

TABLE 1 Anthropometric and clinical variables and acute coronary syndrome (ACS) related risk factors in controls and obstructive sleep apnoea (OSA) patients

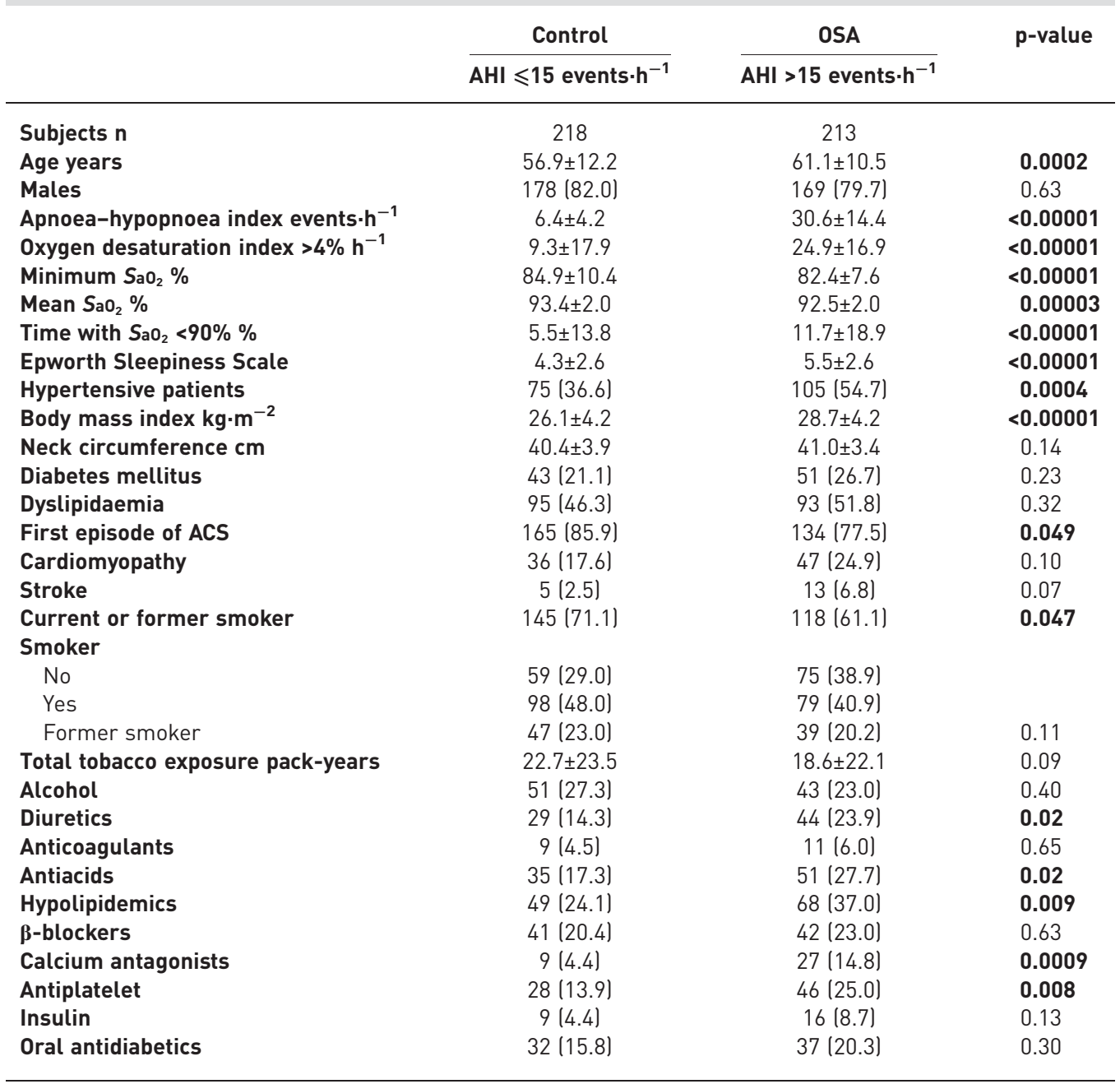

Data are presented as mean \pm SD or $\mathrm{n}(\%)$, unless otherwise stated. $\mathrm{SaO}_{2}$ : arterial oxygen saturation. MannWhitney and Chi-squared tests were used to assess the differences in the means or proportions between groups. Statistically significant $p$-values are shown in bold. 
( $\mathrm{p}=0.0002)$. Hypertension was more prevalent in patients from the OSA group (table 1) $(\mathrm{p}=0.0004)$, who also exhibited an increased use of diuretics and calcium antagonists (table 1) $\mathrm{p}=0.02$ and $\mathrm{p}=0.0009$, respectively). Additionally, OSA patients exhibited a higher BMI and were less likely to be current or former smokers than control patients (table 1) $(\mathrm{p}<0.00001$ and $\mathrm{p}=0.04$, respectively). $95 \%$ of OSA patients and $91 \%$ of controls underwent peripheral coronary intervention, which included $42 \%$ and $44 \%$ undergoing primary peripheral coronary intervention, respectively.

With respect to ACS severity, the percentages of patients with Killip classes II-IV and $\geqslant 3$ diseased vessels were higher in the OSA group (table 2) ( $\mathrm{p}=0.03$ and $\mathrm{p}=0.006$, respectively). For OSA patients, we observed that the mean ejection fraction was lower, whereas the mean peak troponin was higher (table 2) $(\mathrm{p}=0.04$ and $\mathrm{p}=0.005$, respectively). However, the analysis adjusted for smoking, age, BMI and hypertension revealed that only the peak troponin levels were statistically significant (table 2) $(\mathrm{p}=0.03)$.

With respect to the ACS short-term prognosis, the mean length of stay in the coronary care unit was higher in the OSA group even after adjustment (table 3) ( $\mathrm{p}=0.01$ and $\mathrm{p}=0.03)$. Nevertheless, the length of hospitalisation, number of complications and mortality rate during hospitalisation were similar between the groups.

To further investigate the strength of the association of ACS severity-related variables with OSA severity, we classified the patients into four groups according to AHI Q1-4. The results confirmed that the peak troponin levels increased with each AHI Q, even after adjustment (table 4 and fig. 2) ( $\mathrm{p}=0.0003$ and $\mathrm{p}=0.002$ ). Additionally, this analysis revealed that the number of diseased vessels was higher for the most severe OSA group (AHI >27.2 events $\cdot \mathrm{h}^{-1}$ ), even after adjustment (table 4 and fig. 2 ) $(\mathrm{p}=0.001$ and $\mathrm{p}=0.04)$. Moreover, the odds ratios for three or more diseased vessels (with respect to one vessel) were 2.07, 2.08 and 2.36, when comparing individuals above AHI Q1-3 with respect to those below Q2-4, respectively (table S1, $\mathrm{p}=0.11, \mathrm{p}=0.006$ and $\mathrm{p}=0.04$ ). However, when analysing the association between ACS short-term prognosis with OSA severity, there was no statistically significant association (table 5).

Finally, we determined an optimal threshold for each ACS severity outcome to discriminate between OSA and control patients with the goal of obtaining a more detailed description of this association. This analysis demonstrated a significant increase in the risk of an OSA patient to have a peak plasma troponin

TABLE 2 Variables related to acute coronary syndrome (ACS) severity in controls and obstructive sleep apnoea (OSA) patients

\begin{tabular}{|c|c|c|c|c|c|}
\hline & \multirow{2}{*}{$\frac{\text { Control }}{A H I \leqslant 15 \text { events } \cdot h^{-1}}$} & \multirow{2}{*}{$\frac{\text { OSA }}{A H I>15 \text { events } \cdot h^{-1}}$} & \multicolumn{3}{|c|}{$p$-value } \\
\hline & & & A & B & C \\
\hline Subjects $\mathrm{n}$ & 218 & 213 & & & \\
\hline \multicolumn{6}{|l|}{ ACS category } \\
\hline Unstable & 30 (14.8) & 26 (13.3) & & & \\
\hline Non-STEMI & $87(42.6)$ & 81 (41.5) & & & \\
\hline STEMI & $87(42.6)$ & $88(45.2)$ & 0.86 & $0.58^{\#}$ & $0.60^{\#}$ \\
\hline \multicolumn{6}{|l|}{ Killip class } \\
\hline । & 148 (94.3) & 135 (87.7) & & & \\
\hline$\|$ & $7(4.4)$ & $13(8.4)$ & & & \\
\hline III & $2(1.3)$ & $4(2.6)$ & & & \\
\hline IV & $0(0)$ & $2(1.3)$ & 0.17 & $0.03^{\#}$ & $0.30^{\#}$ \\
\hline \multicolumn{6}{|l|}{ Diseased vessels $n$} \\
\hline 1 & 106 (55.8) & $77(43.7)$ & & & \\
\hline 2 & $49(25.8)$ & $45(25.6)$ & & & \\
\hline$\geqslant 3$ & 35 (18.4) & $54(30.7)$ & 0.02 & $0.006 \#$ & $0.12^{\#}$ \\
\hline Stents implanted $\mathrm{n}$ & $1.4 \pm 1.0$ & $1.4 \pm 1.0$ & 0.87 & 0.82 & 0.44 \\
\hline Ejection fraction \% & $57.0 \pm 9.5$ & $54.8 \pm 11.6$ & 0.04 & $0.04^{\pi}$ & $0.17^{\pi}$ \\
\hline Peak troponin $n g \cdot \mathrm{L}^{-1}$ & $831.7 \pm 908.4$ & $987.2 \pm 884.9$ & 0.002 & $0.005^{\pi}$ & $0.03^{\pi}$ \\
\hline
\end{tabular}

Data are presented as $\mathrm{n}(\%)$ or mean \pm SD, unless otherwise stated. AHI: apnoea-hypopnoea index; STEMI: ST-elevation myocardial infarction. p-values, evaluating the differences between groups, were calculated from the following: Mann-Whitney and Chi-squared tests as appropriate (A); regression models (B); regression models adjusted for tobacco (current or former smoker versus nonsmoker), age, body mass index and hypertension (C). \#: ordinal integer values considered in the linear model analyses to evaluate the differences in the trend for the ACS category, Killip classification and number of diseased vessels. ๆ: Log-transformed values considered in linear model analyses for the ejection fraction and peak troponin. Statistically significant $\mathrm{p}$-values are shown in bold. 
TABLE 3 Variables related to the short-term prognosis of acute coronary syndrome (ACS) in controls and obstructive sleep apnoea (OSA) patients

\begin{tabular}{|c|c|c|c|c|c|}
\hline & \multirow{2}{*}{$\frac{\text { Control }}{A H I \leqslant 15 \text { events } \cdot h^{-1}}$} & \multirow{2}{*}{$\frac{\text { OSA }}{A H I>15 \text { events } \cdot h^{-1}}$} & \multicolumn{3}{|c|}{ p-value } \\
\hline & & & A & B & C \\
\hline Subjects $\mathbf{n}$ & 218 & 213 & & & \\
\hline Length of hospitalisation days & $6.5 \pm 3.7$ & $6.8 \pm 3.8$ & 0.4 & 0.55 & 0.68 \\
\hline CV complications during hospitalisation & $14(8.1)$ & $16(9.8)$ & 0.73 & 0.59 & 0.67 \\
\hline Mortality during hospitalisation & $0(0)$ & $1(0.7)$ & $0.29^{\#}$ & $\#$ & $\#$ \\
\hline
\end{tabular}

Data are presented as mean \pm SD or $n(\%)$, unless otherwise stated. AHI: apnoea-hypopnoea index; CCU, coronary care unit; CV: cardiovascular. p-values, to evaluate differences between the groups, were calculated using: Mann-Whitney and Chi-squared tests as appropriate (A); linear and logistic regression models (B); and regression models adjusted by tobacco (current or former smoker versus nonsmoker), age, body mass index and hypertension (C). " : exact test based on the binomial distribution. Logistic regression models were not fit due to the lack of death cases. Statistically significant $\mathrm{p}$-values are shown in bold.

level $>724.5 \mathrm{ng} \cdot \mathrm{L}^{-1}$ (table 6) (OR 2.59, $\mathrm{p}=0.02$ ). This analysis also revealed a significant increase in the risk of an OSA patient to exhibit an ejection fraction lower than 51.5\% (table 6) (OR 2.05, p=0.04) and a tendency to present with more than three diseased vessels (table 6) (OR 1.96, p=0.06).

\section{Discussion}

The results of this observational study suggest that OSA influences the severity of ACS and its short-term prognosis. OSA is related to an increase in peak plasma troponin level, a decrease in the ejection fraction, and an increase in the number of diseased vessels. After adjusting for smoking, hypertension, and BMI, only the peak plasma troponin levels remained significantly related to OSA. The OSA severity, evaluated by $\mathrm{AHI}$, is related to the number of diseased vessels and the peak plasma troponin levels. Patients with

TABLE 4 Variables related to acute coronary syndrome (ACS) severity in control subjects and obstructive sleep apnoea (OSA) patients, considering the apnoea-hypopnoea index (AHI) quartiles (Q)

\begin{tabular}{|c|c|c|c|c|c|c|c|c|}
\hline \multicolumn{4}{|c|}{ AHI events- $\mathrm{h}^{-1}$} & \multicolumn{5}{|c|}{$p$-value } \\
\hline \multirow[t]{2}{*}{ Q1<6 } & Q2 6.1-14.9 & Q3 15-27.2 & Q4 > 27.2 & A & B & & C & \\
\hline & & & & & Qualitative & Ordinal & Qualitative & Ordinal \\
\hline
\end{tabular}

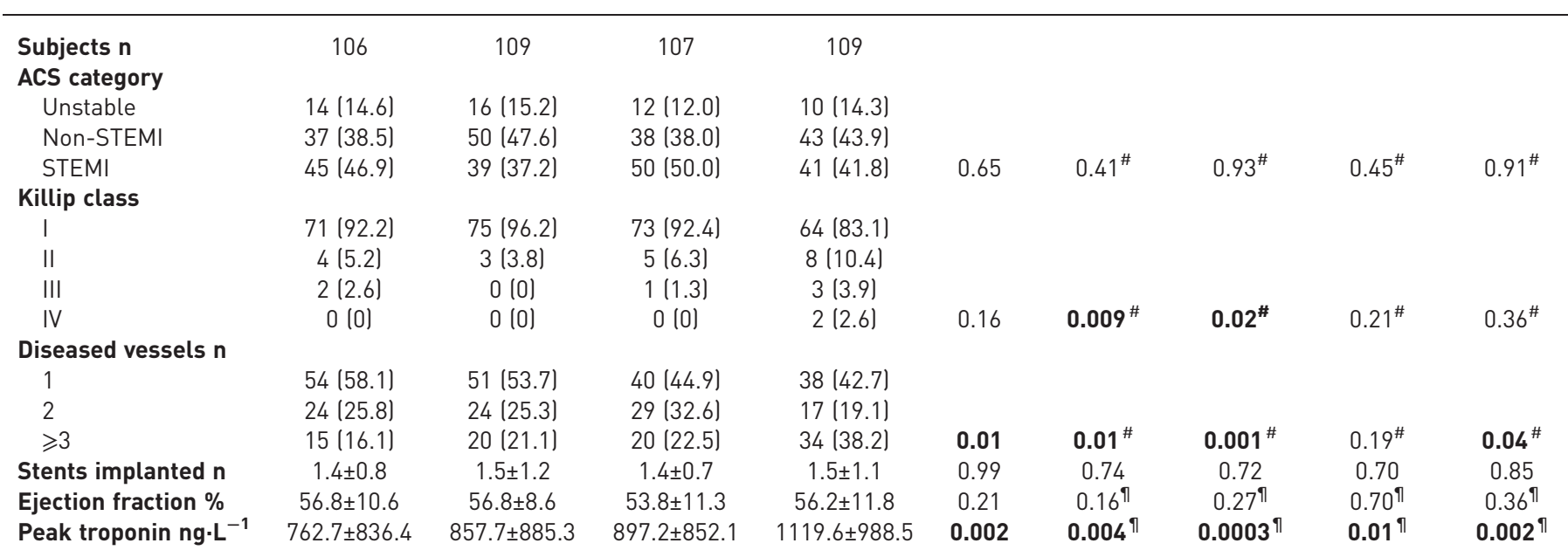

Data are presented as $\mathrm{n}(\%)$ or mean $\pm \mathrm{SD}$, unless otherwise stated. STEMI: ST-elevation myocardial infarction. $\mathrm{p}$-values, to evaluate differences among groups, were computed with: Kruskal-Wallis and Chi-squared tests (A); regression models (B); and regression models adjusted for tobacco (current or former smoker versus nonsmoker), age, body mass index and hypertension (C). \#: ordinal integer values were considered in linear model analyses to evaluate the differences in the trends for the ACS category, Killip classification and number of diseased vessels; ": Log-transformed values were considered in linear model analyses for ejection fraction and peak troponin. In both B and C, p-values from ANOVA tables were obtained when considering the four AHI-based groups, both as qualitative and ordinal integer variables. Statistically significant $\mathrm{p}$-values are shown in bold. 


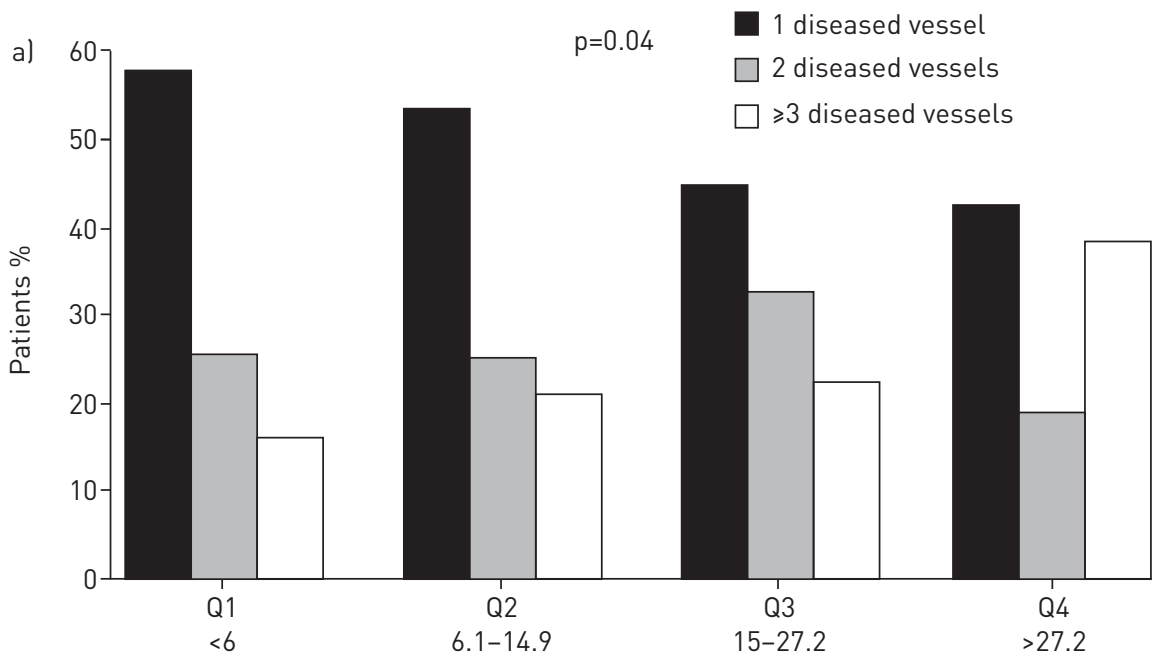

$\mathrm{AHI}$ events $\cdot \mathrm{h}^{-1}$

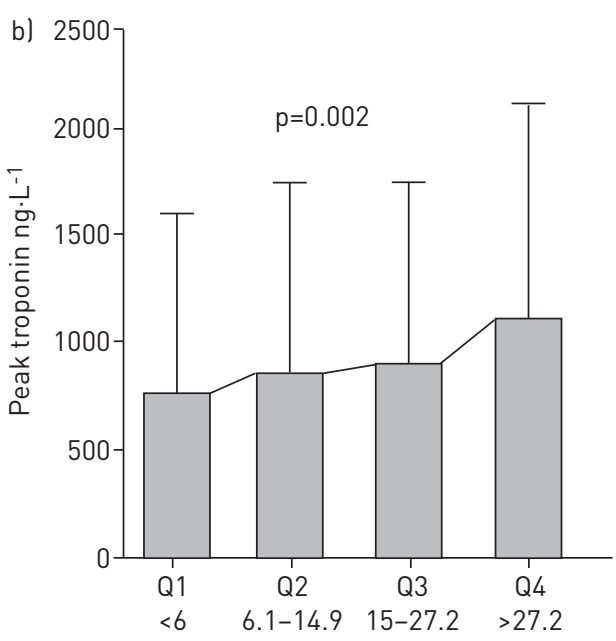

$\mathrm{AHI}$ events $\cdot \mathrm{h}^{-1}$

FIGURE 2 Association between acute coronary syndrome severity variables and obstructive sleep apnoea. a) Frequency distribution for the number of diseased vessels in apnoea-hypopnoea index (AHI) quartiles (Q). b) Peak troponin mean values for AHI Q1-4, segments represent one standard deviation. The analysis was adjusted by smoking, age, body mass index, and hypertension. The adjusted p-values in table 4 are shown.

OSA spent more time in the coronary care unit, although the length of hospitalisation was similar between patients with and without OSA.

Available data from clinic-based cohorts suggest that OSA is an independent risk factor for myocardial infarction and other coronary events [3, 18-21]. Furthermore, OSA worsens the long-term prognosis of ACS [22, 23]. Several pathogenic factors are proposed as intermediate mechanisms linking OSA with cardiovascular disease [5]. It has been described that systemic inflammation induced by chronic intermittent hypoxia may play a key role in atherogenesis in OSA patients [24]. These intermediate mechanisms can produce cardiac hyper-excitability and increase the sympathetic tone, oxidative stress and hypercoagulability, which could in turn increase the severity of ACS in OSA patients and worsen the prognosis of ACS. Additionally, chronic intermittent hypoxia increases the infarct size in animal models [25]. Also, the deleterious effects of sleep disordered breathing in infarct expansion and impaired healing of myocardial tissue and coronary artery plaque burden have been described [26-28]. The increased number of affected vessels and the increase in the peak plasma troponin levels in the group of patients with OSA could be associated with worse long-term prognosis. In contrast to our results, LEE et al. [29] reported similar severity in acute myocardial infarction in patients with and without OSA. This study also demonstrated that there was no significant association between OSA and impaired microvascular perfusion after primary percutaneous coronary intervention. Interestingly, SHAH et al. [12] explained that patients with OSA exhibit less severe cardiac injuries during an acute nonfatal myocardial infarction compared

Table 5 Variables related to acute coronary syndrome short-term prognosis in control subjects and obstructive sleep apnoea

\begin{tabular}{|c|c|c|c|c|c|c|c|c|}
\hline \multicolumn{4}{|c|}{ AHI events: $h^{-1}$} & \multicolumn{5}{|c|}{$p$-value } \\
\hline Q1 $<6$ & Q2 6.1-14.9 & Q3 15-27.2 & $Q 4>27.2$ & A & B & & C & \\
\hline & & & & & Qualitative & Ordinal & Qualitative & Ordinal \\
\hline
\end{tabular}

\begin{tabular}{|c|c|c|c|c|c|c|c|c|c|}
\hline Subjects $n$ & 106 & 109 & 107 & 109 & & & & & \\
\hline Length of stay in the CCU days & $2.3 \pm 1.1$ & $2.3 \pm 0.8$ & $2.6 \pm 1.4$ & $2.6 \pm 1.1$ & 0.25 & 0.12 & 0.06 & 0.19 & 0.14 \\
\hline Length of hospitalisation days & $6.6 \pm 3.8$ & $6.5 \pm 3.7$ & $7.0 \pm 4.4$ & $6.5 \pm 3.0$ & 0.88 & 0.80 & 0.88 & 0.72 & 0.81 \\
\hline $\begin{array}{l}\text { CV complications during } \\
\text { hospitalisation }\end{array}$ & $7(8.3)$ & $7(8.1)$ & $10(11.6)$ & $6(7.4)$ & 0.78 & 0.79 & 0.94 & 0.68 & 0.43 \\
\hline
\end{tabular}

Data are presented as mean \pm SD or $\mathrm{n}(\%)$, unless otherwise stated. Q: quartile; $\mathrm{AHI}$ : apnoea-hypopnoea index; CCU, coronary care unit; CV: cardiovascular. p-values to evaluate differences among groups were computed with: Kruskal-Wallis and Chi-squared tests (A); linear and logistic regression models (B); and regression models adjusted for tobacco (current or former smoker versus nonsmoker), age, body mass index and hypertension (C). In both B and C the p-values from ANOVA or deviance tables were obtained when considering the fourth AHI-based groups, both as qualitative and ordinal integer variables. 
Table 6 Variables related to acute coronary syndrome severity in controls and obstructive sleep apnoea (OSA) patients, considering an optimal threshold for the responses

\begin{tabular}{|c|c|c|c|}
\hline & \multicolumn{2}{|c|}{ Univariate model } & \multirow{2}{*}{$\frac{\text { Adjusted model }}{\mathrm{p} \text {-value }}$} \\
\hline & OR $(95 \% \mathrm{CI})$ & p-value & \\
\hline \multicolumn{4}{|c|}{ Ejection fraction $<51.5 \%$} \\
\hline Control & 1 & & \\
\hline OSA & $2.05(1.24-3.43)$ & 0.005 & 0.04 \\
\hline \multicolumn{4}{|c|}{ Killip classification >1 } \\
\hline Control & 1 & & \\
\hline OSA & $2.31(1.04-5.53)$ & 0.046 & 0.17 \\
\hline \multicolumn{4}{|c|}{$\geqslant 3$ diseased vessels } \\
\hline Control & 1 & & \\
\hline OSA & $1.96(1.21-3.21)$ & 0.007 & 0.06 \\
\hline \multicolumn{4}{|c|}{ Peak troponin $\geqslant 724.5 \mathrm{ng} \cdot \mathrm{mL}^{-1}$} \\
\hline Control & 1 & & \\
\hline OSA & $2.59(1.53-4.46)$ & $<0.001$ & 0.02 \\
\hline \multicolumn{4}{|c|}{$\begin{array}{l}\text { The Gini criterion was used to determine an optimal threshold to discriminate between OSA and control } \\
\text { patients for each variable. The odds ratio (OR) and the corresponding } 95 \% \text { confidence interval (CI) and } \\
\text { p-values estimated from logistic regression models. p-values from the logistic regression models adjusted } \\
\text { for tobacco (current or former smoker versus nonsmoker), age, body mass index and hypertension are also } \\
\text { provided. Only significant variables identified in table } 2 \text { were considered. Statistically significant p-values } \\
\text { are shown in bold. }\end{array}$} \\
\hline
\end{tabular}

with patients without OSA. The authors suggest a cardioprotective role of OSA during acute myocardial infarction induced by ischaemic preconditioning. BERGer et al. [11] demonstrate that patients with AMI, who exhibit mild-to-moderate sleep disordered breathing, can activate adaptive mechanisms and may improve endothelial function and provide cardioprotection in the context of acute myocardial infarction. Additionally, organ autoregulation could be a mechanism implicated in maintaining a constant blood flow during fluctuations in its perfusion pressure in patients with ACS [30].

The strengths of our study include its multicentre design with a large number of patients. All participating centres performed the same methodology and the sleep study was performed with the same model of polygraph. Nevertheless, this study has several potential limitations. First, we excluded patients with the more severe ACS and worse prognosis (cardiogenic shock). Additionally, we excluded patients with daytime sleepiness who exhibited the most severe OSA. Nevertheless, the number of excluded patients for these causes was relatively low. Secondly, subjects in this study were predominantly male, therefore, the results cannot be extrapolated to females. Thirdly, the diagnosis is based on respiratory polygraphy, which could underestimate the severity of OSA. However, due to the critical situation of the patients, full polysomnography monitoring could be a stressful procedure for this high-risk patient group. Finally, the design of this study does not allow us to draw definitive conclusions and further randomised control trials on this topic will be necessary.

\section{Conclusions}

The results of this study indicate that OSA is correlated with an increase in the peak plasma troponin levels, number of diseased vessels, and length of stay in the coronary unit. These correlations may have both short- and long-term consequences. Large-scale randomised control trials are urgently needed to evaluate interventions in this setting.

\section{References}

1 Durán J, Esnaola S, Rubio R, et al. Obstructive sleep apnea-hypopnea and related clinical features in a population-based sample of subjects aged 30 to 70 yr. Am J Respir Crit Care Med 2001; 163: 685-689.

2 Peppard PE, Young T, Barnet JH, et al. Increased prevalence of sleep-disordered breathing in adults. Am J Epidemiol 2013; 177: 1006-1014.

3 Marin JM, Carrizo SJ, Vicente E, et al. Long-term cardiovascular outcomes in men with obstructive sleep apnoea-hypopnoea with or without treatment with continuous positive airway pressure: an observational study. Lancet 2005; 365: 1046-1053.

4 Barbé F, Durán-Cantolla J, Sánchez-de-la-Torre M, et al. Effect of continuous positive airway pressure on the incidence of hypertension and cardiovascular events in nonsleepy patients with obstructive sleep apnea: a randomized controlled trial. JAMA 2012; 307: 2161-2168. 
5 Sánchez-de-la-Torre M, Campos-Rodriguez F, Barbé F, et al. Obstructive sleep apnoea and cardiovascular disease Lancet Respir Med 2013; 1: 61-72.

6 Kohler M, Stradling JR. Mechanisms of vascular damage in obstructive sleep apnea. Nat Rev Cardiol 2010; 7: 677-685.

7 Somers VK, Dyken ME, Clary MP, et al. Sympathetic neural mechanisms in obstructive sleep apnea. J Clin Invest 1995; 96: 1897-1904.

8 Gami AS, Olson EJ, Shen WK, et al. Obstructive sleep apnea and the risk of sudden cardiac death: a longitudinal study of 10,701 adults. J Am Coll Cardiol 2013; 62: 610-616.

9 Harbison J, O’Reilly P, McNicholas WT. Cardiac rhythm disturbances in the obstructive sleep apnea syndrome: effects of nasal continuous positive airway pressure therapy. Chest 2000; 118: 591-595.

10 Gami AS, Howard DE, Olson EJ, et al. Day-night pattern of sudden death in obstructive sleep apnea. N Engl J Med 2005; 352: 1206-1214.

11 Berger S, Aronson D, Lavie P, et al. Endothelial progenitor cells in acute myocardial infarction and sleep-disordered breathing. Am J Respir Crit Care Med 2013; 187: 90-98.

12 Shah N, Redline S, Yaggi HK, et al. Obstructive sleep apnea and acute myocardial infarction severity: ischemic preconditioning?. Sleep breath 2013; 17: 819-826.

13 Esquinas $\mathrm{C}$, Sánchez-de-la-Torre $\mathrm{M}$, Aldomá $\mathrm{A}$, et al. Rationale and methodology of the impact of continuous positive airway pressure on patients with ACS and nonsleepy OSA: the ISAACC Trial. Clin Cardiol 2013; 36: 495-501.

14 Deckers JW. Classification of myocardial infarction and unstable angina: a re-assessment. Int J Cardiol 2013; 167: $2387-2390$

15 Spanish National Consensus in Sleep Apnea-Hypopnea Syndrome (SAHS). Arch Bronconeumol 2005; 41: Suppl. 4 , 3-11.

16 Chiner E, Arriero JM, Signes-Costa J, et al. Validación de la versión española del test de somnolencia Epworth en pacientes con síndrome de apnea de sueno [Validation of the Spanish version of the Epworth Sleepiness Scale in patients with a sleep apnea syndrome]. Arch Bronconeumol 1999; 35: 422-427.

17 R Core Team. R A. Language and environment for statistical computing. R Foundation for Statistical Computing, Vienna, 2013. www.R-project.org/ Date last accessed:. November 4:2014

18 Peker Y, Carlson J, Hedner J. Increased incidence of coronary artery disease in sleep apnoea: a long-term follow-up. Eur Respir J 2006; 28: 596-602.

19 Schäfer H, Koehler U, Ewig S, et al. Obstructive sleep apnea as a risk marker in coronary artery disease. Cardiology 1999; 92: 79-84.

20 Shah NA, Yaggi HK, Concato J, et al. Obstructive sleep apnea as a risk factor for coronary events or cardiovascular death. Sleep Breath 2010; 14: 131-136.

21 Shahar E, Whitney CW, Redline S, et al. Sleep-disordered breathing and cardiovascular disease: cross-sectional results of the Sleep Heart Health Study. Am J Respir Crit Care Med 2001; 163: 19-25.

22 Peker Y, Hedner J, Kraiczi H, et al. Respiratory disturbance index: an independent predictor of mortality in coronary artery disease. Am J Respir Crit Care Med 2000; 162: 81-86.

23 Mooe T, Franklin KA, Holmström K, et al. Sleep-disordered breathing and coronary artery disease: long-term prognosis. Am J Respir Crit Care Med 2001; 164: 1910-1913.

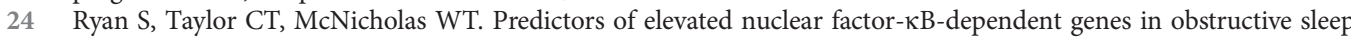
apnea syndrome. Am J Respir Crit Care Med 2006; 174: 824-830.

25 Belaidi E, Joyeux-Faure M, Ribuot C, et al. Major role for hypoxia inducible factor-1 and the endothelin system in promoting myocardial infarction and hypertension in an animal model of obstructive sleep apnea. J Am Coll Cardiol 2009; 53: 1309-1317.

26 Buchner S, Satzl A, Debl K, et al. Impact of sleep-disordered breathing on myocardial salvage and infarct size in patients with acute myocardial infarction. Eur H J 2014; 35: 192-199.

27 Sorajja D, Gami AS, Somers VK, et al. Independent association between obstructive sleep apnea and subclinical coronary artery disease. Chest 2008; 133: 927-933.

28 Kent BD, Garvey JF, Ryan S, et al. Severity of obstructive sleep apnoea predicts coronary artery plaque burden: a coronary computed tomographic angiography study. Eur Respir J 2013; 42: 1263-1270.

29 Lee CH, Khoo SM, Tai BC, et al. Obstructive sleep apnea in patients admitted for acute myocardial infarction: prevalence, predictors, and effect on microvascular perfusion. Chest 2009; 135: 1488-1495.

30 Urbano F, Roux F, Schindler J, et al. Impaired cerebral autoregulation in obstructive sleep apnea. J Appl Physiol 2008; 105: 1852-1857. 